\title{
Deformed Gaussian orthogonal ensemble and the statistical fluctuations in the spectra of the quartic oscillator
}

\author{
C. I. Barbosa* \\ Instituto de Física Teórica Universidade Estadual Paulista, Rua Pamplona 145, 01405-900 São Paulo, SP, Brazil \\ C. L. Lima, M. S. Hussein, and M. P. Pato \\ Nuclear Theory and Elementary Particle Phenomenology Group, Instituto de Física, Universidade de São Paulo, Caixa Postal 66318, \\ 05389-970 São Paulo, SP, Brazil \\ (Received 29 January 1998)
}

\begin{abstract}
The nearest-neighbor spacing distributions proposed by four models, namely, the Berry-Robnik, CaurierGrammaticos-Ramani, Lenz-Haake, and the deformed Gaussian orthogonal ensemble, as well as the ansatz by Brody, are applied to the transition between chaos and order that occurs in the isotropic quartic oscillator. The advantages and disadvantages of these five descriptions are discussed. In addition, the results of a simple extension of the expression for the Dyson-Mehta statistic $\Delta_{3}$ are compared with those of a more popular one, usually associated with the Berry-Robnik formalism. [S1063-651X(99)01201-5]

PACS number(s): 05.45.Xt, 47.52. $+\mathrm{j}, 24.60 .-\mathrm{k}$
\end{abstract}

\section{INTRODUCTION}

Thanks to the pioneering work of Berry and Tabor [1] and to the work of Bohigas, Giannoni, and Schmit [2], it is nowadays largely accepted that regular systems show level fluctuations of the Poissonian type while chaotic systems have eigenvalue fluctuations like those of the matrix ensembles of Dyson. Particularly, in the case of the nearest-neighbor spacing distribution (NND), the Poissonian case reads

$$
P_{\mathrm{Poi}}(s)=e^{-s}
$$

whereas the so-called Wigner distribution is

$$
P_{\mathrm{GOE}}(s) \sim P_{\mathrm{Wigner}}(s)=\frac{\pi}{2} s \exp \left(\frac{-\pi}{4} s^{2}\right),
$$

where $s$ is the spacing between adjacent levels. This expression is obtained for an ensemble of two-dimensional matrices with $\langle s\rangle=1$. This result is very near to that obtained in the limit of a very large dimension [3].

The description of systems intermediate between chaos and order has been largely discussed in the literature, and many models were proposed. Some of the ideas used to describe this transition are represented by four models: the Berry-Robnik model [4] based on semiclassical arguments, the $2 \times 2$ matrix model by Caurier, Grammaticos, and Ramani (CGR) [5], the Lenz-Haake (LH) model [6], a $2 \times 2$ matrix model based on Dyson's proposal [7], and finally the deformed Gaussian orthogonal ensemble (DGOE) [8] which uses both concepts of information theory and Dyson's proposal to construct an ensemble of $N \times N$ matrices. The purpose of the present paper is to compare these four models and the Brody ansatz with each other. In this way, their NND's were applied to the isotropic quartic oscillator and

\footnotetext{
*Present address: Max-Planck-Institut für Kernphysik, D-69029 Heidelberg, Germany.
}

the merits and difficulties of these descriptions were discussed. In addition, long range correlations are analyzed in terms of the Dyson-Mehta statistic $\Delta_{3}(L)$.

The paper is organized as follows: in Sec. II, the main ideas and the NND of each description, as well as the expressions used to fit $\Delta_{3}(L)$, are presented. In Sec. III, the main features of the quartic oscillator are summarized. In Sec. IV, the analysis and results are presented. A short discussion in Sec. V concludes the paper.

\section{MODELS}

The first widely used expression for the NND of intermediate systems was proposed by Brody [9] in 1973 with the aim of quantifying the repulsion among energy levels [10]. He suggested the ansatz

$$
P_{\text {Brody }}(s)=A s^{\omega} \exp \left(-\alpha s^{w+1}\right)
$$

where $A$ and $\alpha$ depend on $\omega$ in the following ways:

$$
\begin{gathered}
A=(\omega+1) \alpha, \\
\alpha=\left[\Gamma\left(\frac{\omega+2}{\omega+1}\right)\right]^{\omega+1} .
\end{gathered}
$$

It is easy to see that when $\omega=0$, no level repulsion exists, and this gives the Poisson distribution; when $\omega=1$ the Wigner distribution is obtained. In addition, Eq. (4) guarantees the proper normalization of $P_{\text {Brody }}(x)$. This handy expression has turned the parameter $\omega$ in Eq. (3) into a widely used gauge of the chaoticity of intermediate systems, although it lacks physical meaning. As a matter of fact, other models were proposed since then aiming at a more physical description of the chaos-order transition. 
In 1984, Berry and Robnik proposed a model based on semiclassical arguments [4]. By considering the phase space as made up of statistically independent regular and chaotic parts, they found

$$
\begin{aligned}
P_{B R}(s)= & \rho^{2} e^{-\rho s} \operatorname{erfc}\left(\frac{\sqrt{\pi}}{2}(1-\rho) s\right) \\
& +\left[2 \rho(1-\rho)+\frac{1}{2} \pi(1-\rho)^{3} s\right] \\
& \times \exp \left(-\rho s-\frac{1}{4}(1-\rho)^{2} s^{2}\right),
\end{aligned}
$$

which interpolates between the Wigner and Poisson distributions when $\rho$ goes from zero to unity. Although good results were obtained, the statistical independence of the chaotic and regular parts of the phase space implies that there is no level repulsion in the intermediate region between chaos and order. However, it is physically expected that level repulsion persists over a sufficiently short level distance $s$ until the pure Poissonian case is reached [10].

The model proposed in 1990 by Caurier, Grammaticos, and Ramani [5] uses an ensemble of $2 \times 2$ matrices, where the off-diagonal term is modulated as a perturbation. They found

$$
P_{\mathrm{CGR}}(s)=\frac{s}{\gamma} \sqrt{\frac{\pi}{8}} I_{0}\left(\frac{s^{2}}{16 \gamma^{2}}\right) \exp \left(-\frac{s^{2}}{16 \gamma^{2}}\right) e^{-s}
$$

The Poissonian situation is obtained when $\gamma=0$. As $\gamma$ increases, expression (6) tends to the Wigner distribution, however, it leads to numerical problems when approaching that limit.

A more sophisticated $2 \times 2$ matrix model was proposed in 1996 by Lenz and Haake [6] by considering Dyson's proposal [7]. This says that any mixed system can be described by the Hamiltonian

$$
H=H_{0}+\varepsilon H_{1},
$$

where $H_{0}$ is the Hamiltonian which describes the original Poissonian situation, and $H_{1}$ provides the coupling between the levels defined by $H_{0}$; in the case of strongest coupling between $H_{0}$ and $H_{1}$, the Hamiltonian $H$ will be a GOE matrix. By working out the related joint probability distribution $P[H]$ of the matrix elements, the authors were able to write the NND of an ensemble of $2 \times 2$ matrices as

$$
\begin{aligned}
P_{L H}(s)= & \frac{s u(\lambda)^{2}}{\lambda} \exp \left(\frac{-u(\lambda)^{2} s^{2}}{4 \lambda^{2}}\right) \\
& \times \int_{0}^{\infty} e^{-\left(\xi^{2}+2 \xi \lambda\right)} I_{0}\left(\frac{s \xi u(\lambda)}{\lambda}\right) d \xi .
\end{aligned}
$$

Here

$$
u(\lambda)=\exp \left(\frac{\lambda^{2}}{2}\right) \int_{0}^{\pi / 2} \cos \left[\frac{\lambda^{2}}{2} \tan \theta-\theta\right] d \theta
$$

is the Kummer function, $I_{0}$ is the modified Bessel function of zeroth order [11]. For this Lenz-Haake distribution the Poissonian case is achieved when $\lambda=0$, and, for large enough $\lambda$, the Wigner distribution is obtained. In fact, for $\lambda=1$, Eq. (8) is already very similar to the Wigner distribution.

Despite the good results generally obtained by the above models in the description of NND fluctuations, many aspects of systems in the transition between chaos and order are not properly described. For example, these models do not describe the statistical fluctuations of the eigenvector coefficients or the long range correlations among levels. In the case of matrix models like those of Caurier, Grammaticos, and Ramani and Lenz and Haake, the description of such features requires a joint probability distribution $P[H]$ for high-dimensional matrices. This cannot be calculated in these two models, since they are based on ensembles of 2 $\times 2$ matrices. On the other hand, the simplification of considering the chaotic and regular parts of phase space as statistically independent-proposed by Berry and Robnikallows one to use the sum property of the Dyson-Mehta statistic $\Delta_{3}$, a typical measure of long range correlations [3]; that is

$$
\Delta_{3}^{\mathrm{BR}}(L)=\Delta_{3}^{\mathrm{GOE}}[\rho L]+\Delta_{3}^{\mathrm{Poi}}[(1-\rho) L]
$$

Here $L$ is an energy interval in units of the mean level distance, and $\rho$ is a measure of the chaotic fraction of phase space [see Eq. (5)]. The Poissonian term reads

$$
\Delta_{3}^{\mathrm{Poi}}(L)=\frac{L}{15}
$$

and the GOE term is

$$
\Delta_{3}^{\mathrm{GOE}}(L)=\int_{0}^{L} d r \frac{2}{L^{4}}\left(L^{3}-2 L^{2} r+r^{3}\right) \Sigma^{2}(r) .
$$

In this expression, $\Sigma^{2}$ is the so-called $\Sigma^{2}$ statistic or number variance. It is the average squared difference $\Sigma^{2}(L)$ $=\left\langle[n(L)-L]^{2}\right\rangle$ between the actual number $n(L)$ and the expected number $L$ of levels in an interval of given length; see Ref. [3]. Expression (10) gives good results when describing data [12], but it is important to keep in mind that the statistical independence proposed in this framework has the undesirable consequence of eliminating the level repulsion in the transition region between chaos and order, as discussed above.

In order to avoid these problems, two of us proposed a matrix model [8] which also makes use of Dyson's proposal, but now written in terms of high-dimensional matrices. The ensemble proposed in Ref. [8] was named the "deformed Gaussian orthogonal ensemble,', where the term "deformed" is due to Dyson [10]. The difficulties with the analytical calculation of a joint probability distribution for highdimensional matrices are avoided by using information theory in a similar way as in Balian's work [13]. The information content of an ensemble of matrices is defined as

$$
I\{P[H]\}=\int d[H] P[H] \ln P[H],
$$


where

$$
\begin{aligned}
P_{\mathrm{DGOE}}[H]= & P_{\mathrm{GOE}}[H](1+\alpha)^{M(N-M) / 2} \\
& \times \exp \left(\alpha \sum_{i} \operatorname{Tr} P H Q H P\right),
\end{aligned}
$$

The desired joint probability distribution is obtained by the minimization of $I\{P[H]\}$ subject to appropriate constraints [14].

Two of these constraints are

$$
\begin{gathered}
\int d[H] P[H]=1, \\
\left\langle\operatorname{Tr} H^{2}\right\rangle=\int d[H] P[H] \operatorname{Tr} H^{2}=C_{1},
\end{gathered}
$$

the first of which requires the usual normalization, and the second of which limits the length of the spectrum of $H$. By minimizing expression (13) subject to these two constraints, the GOE distribution is obtained.

In order to construct an ensemble of matrices able to describe the level fluctuations of systems in the transition region between chaos and order, it is necessary to introduce a third constraint. This was done in Ref. [8], and here we will reproduce the arguments presented there to explain the constraint.

First let us introduce the projection operators

$$
P=\sum_{i=1}^{M}|i\rangle\langle i|, \quad Q=1-P,
$$

where $|i\rangle$ are basis vectors chosen such that $P$ and $Q$ project $H$ on subspaces that belong to different symmetry classes, as e.g., different parities. By help of the projection operators, the Hamiltonians $H$ are decomposed into blocks

$$
H=\left(\begin{array}{cc}
P H P & P H Q \\
Q H P & Q H Q
\end{array}\right)=V_{0}+V_{1} \text {. }
$$

$V_{0}$ and $V_{1}$ are matrices for which just the diagonal respectively nondiagonal blocks are nonzero.

We impose the condition that the variance of the diagonal and off-diagonal blocks be different, and that this difference be controlled by an additional parameter. This condition expresses Dyson's proposal.

By properly choosing $V_{0}$ and $V_{1}$ the transition between the GOE and the Poisson limits can be described. The third condition is formalized by

$$
\langle\operatorname{Tr} P H Q H P\rangle=\sum_{i=1}^{M} \int d[H] P[H] \operatorname{Tr} P H Q H P=C_{2},
$$

By using Lagrange multipliers it is now possible to calculate the desired joint probability distribution. For convenience one defines a new parameter $\alpha$ as the ratio between $C_{1}$ and $C_{2}$. In this way one obtains where

$$
P_{\mathrm{GOE}}[H]=2^{-N / 2}\left(\frac{\pi}{2}\right)^{-N(N-1) / 4} \exp \left(-\operatorname{Tr} H^{2}\right) .
$$

Here $M$ is the dimension of the blocks and $N$ is the dimension of the entire matrix. By taking $N=2$ and $M=1$, it is possible to obtain an expression to describe the NND for the DGOE:

$$
P_{\mathrm{DGOE}}(s)=\left(1+\frac{\alpha}{2}\right)^{1 / 2} s \exp \left\{-\left(\frac{1}{2}+\frac{\alpha}{8}\right) s^{2}\right\} I_{0}\left(\frac{\alpha s^{2}}{8}\right) .
$$

The function $I_{0}(x)$ is the modified Bessel function of zeroth order [11]. Here the Wigner limit is obtained when $\alpha=0$, and the Poisson distribution when $\alpha \rightarrow \infty$. It is important to note that although this expression was obtained for $2 \times 2$ matrices, Eq. (18) was obtained for an arbitrary dimension. This opened the possibility of studying the statistical fluctuations of the eigenvector coefficients [15]. Another consequence of Eq. (18) is the possibility to study the long range correlations among levels, for example, using the above $\Delta_{3}$ statistic. Unfortunately, an analytical expression for $\Delta_{3}(L)$ obtained in the DGOE framework is not yet available.

A simple ansatz for the Dyson-Mehta statistic which interpolates between the Poissonian and Gaussian limits is

$$
\Delta_{3}^{\mathrm{ansatz}}(L)=q \Delta_{3}^{\mathrm{GOE}}(L)+(1-q) \Delta_{3}^{\mathrm{Poi}}(L) .
$$

This expression was used in the analysis of the eigenvalues of the isotropic quartic oscillator and the results were compared with those from Eq. (10). Equation (21) is just an empirical linear interpolation; therefore, the $q$ parameter here - contrary to $\rho$ in the $\Delta_{3}^{\mathrm{BR}}$ case - is not a measure of the fraction of phase space that is chaotic.

\section{ISOTROPIC QUARTIC OSCILLATOR}

The isotropic quartic oscillator is a system described by the classical Hamiltonian

$$
H=\frac{\left(p_{x}^{2}+p_{y}^{2}\right)}{2}+\frac{x^{2} y^{2}}{2}+\frac{\beta\left(x^{4}+y^{4}\right)}{4},
$$

and the quantum analog is

$$
\left\{-\frac{\hbar^{2}}{2} \nabla+\frac{x^{2} y^{2}}{2}+\frac{\beta\left(x^{4}+y^{4}\right)}{4}\right\} \psi=E \psi .
$$

This system is interesting for the comparison of the five descriptions cited in Sec. II, since it shows a well studied transition between chaos and order (see, for example, Ref. [16]) when $\beta$ goes from zero to 1 . Although there is no physical system described by this Hamiltonian, its dynamical properties may be important for many physical areas as for example in QCD, thanks to the functional similarity with the 
Yang-Mills equations $[17,18]$. Our aim is to compare the above five descriptions in the case of situations intermediate between chaos and order for this quartic oscillator. The quartic oscillator itself-i.e., the relationship between the parameter $\beta$ of Hamiltonian (23) and the chaoticity of the system, the question how fast the transition between chaos and order occurs, whether this transition is monotonic with $\beta$, etc.- -all this is not investigated here. These questions have been discussed in many other works (see Refs. $[16,17]$ and references therein).

An important feature of this system is the scaling between orbits at different energies. By considering a trajectory $(p(t), q(t))$ at energy $E$, it is possible to determine another trajectory $\left(p_{0}(t), q_{0}(t)\right)$ at energy $E_{0}$ by the scaling

$$
p(t)=\varepsilon^{2 / 3} p_{0}\left(\varepsilon^{3} t\right), \quad q(t)=\varepsilon^{1 / 3} q_{0}\left(\varepsilon^{3} t\right),
$$

where $\varepsilon=\left(E / E_{0}\right)^{3 / 4}$ is the scaling factor between different trajectories. The energy $E_{0}$ is taken to be 0.5 . This means that the ground state energy is approximately equal to unity.
TABLE I. Limiting parameter values of DGOE, CaurierGrammaticos-Ramani (CGR), Lenz-Haake (LH), Brody, and BerryRobnik (BR) for the numerical fitting (maximum chaoticity-GOE; maximum regularity-Poisson).

\begin{tabular}{lcc}
\hline \hline Model & GOE & Poisson \\
\hline DGOE & 0 & 500 \\
CGR & 1 & 0 \\
LH & 1 & 0 \\
Brody & 1 & 0 \\
BR & 0 & 1 \\
\hline \hline
\end{tabular}

The average behavior of the cumulative level density $\overline{N(E)}$ could not be written in closed form due to analytical difficulties related to the isotropy property of the Hamiltonian [19]. Nevertheless, it is known [19] that $\overline{N(E)}$ must be proportional to the scaling factor up to second order, namely,

$$
\overline{N(E)} \propto E^{3 / 2} \propto \varepsilon^{2} .
$$

TABLE II. Results from the NND of the isotropic quartic oscillator. Under the heading "Class," the symmetry class and the parameter $\beta$ appearing in the Hamiltonian (23) are listed. For the five descriptions DGOE, Caurier-Grammaticos-Ramani (CGR), Lenz-Haake (LH), Brody, and Berry-Robnik (BR), the parameters of the NND are given together with the associated error $(\sigma)$ and the normalized $\overline{\chi^{2}}$ of the

\begin{tabular}{|c|c|c|c|c|c|c|c|c|c|c|}
\hline \multirow[b]{2}{*}{ Class } & \multicolumn{2}{|l|}{ DGOE } & \multicolumn{2}{|c|}{ CGR } & \multicolumn{2}{|l|}{ LH } & \multicolumn{2}{|c|}{ Brody } & \multicolumn{2}{|l|}{$\mathrm{BR}$} \\
\hline & $\alpha \pm \sigma_{\alpha}$ & $\overline{\chi^{2}}$ & $\gamma \pm \sigma_{\gamma}$ & $\overline{\chi^{2}}$ & $\lambda \pm \sigma_{\lambda}$ & $\overline{\chi^{2}}$ & $\omega \pm \sigma_{\omega}$ & $\overline{\chi^{2}}$ & $\rho \pm \sigma_{\rho}$ & $\overline{\chi^{2}}$ \\
\hline $\mathrm{A} 1, \beta=0.01$ & $3.44 \pm 0.19$ & $(1.4)$ & $0.35 \pm 0.01$ & $(5.0)$ & $0.62 \pm 0.14$ & $(1.6)$ & $0.83 \pm 0.07$ & $(1.2)$ & $0.06 \pm 0.03$ & (1.1) \\
\hline $\mathrm{A} 1, \beta=0.2$ & $9.31 \pm 0.15$ & $(3.7)$ & $0.23 \pm 0.01$ & $(3.2)$ & $0.29 \pm 0.02$ & $(2.4)$ & $0.48 \pm 0.03$ & $(0.9)$ & $0.22 \pm 0.02$ & (1.5) \\
\hline $\mathrm{A} 1, \beta=0.4$ & $500.00 \pm 0.15$ & $(3.7)$ & $0.23 \pm 0.01$ & $(4.9)$ & $0.26 \pm 0.03$ & $(6.2)$ & $0.13 \pm 0.02$ & $(0.8)$ & $0.52 \pm 0.02$ & $(0.5)$ \\
\hline $\mathrm{A} 1, \beta=0.6$ & $500.00 \pm 0.15$ & (3.9) & $0.17 \pm 0.01$ & $(4.5)$ & $0.26 \pm 0.03$ & (6.4) & $0.15 \pm 0.03$ & (1.3) & $0.50 \pm 0.02$ & (1.0) \\
\hline $\mathrm{A} 1, \beta=0.8$ & $500.00 \pm 0.16$ & $(4.2)$ & $0.28 \pm 0.01$ & $(5.5)$ & $0.27 \pm 0.03$ & $(6.0)$ & $0.14 \pm 0.03$ & (1.1) & $0.54 \pm 0.02$ & $(0.8)$ \\
\hline $\mathrm{A} 1, \beta=1.0$ & $500.00 \pm 0.17$ & $(5.4)$ & $0.31 \pm 0.01$ & $(6.8)$ & $0.25 \pm 0.03$ & $(7.3)$ & $0.10 \pm 0.03$ & (1.8) & $0.64 \pm 0.02$ & (1.8) \\
\hline $\mathrm{B} 2, \beta=0.01$ & $3.12 \pm 0.08$ & $(0.2)$ & $0.35 \pm 0.02$ & $(4.8)$ & $0.76 \pm 0.10$ & $(0.5)$ & $0.91 \pm 0.03$ & $(0.2)$ & $0.03 \pm 0.01$ & $(0.2)$ \\
\hline $\mathrm{B} 2, \beta=0.2$ & $24.59 \pm 0.11$ & (2.4) & $0.29 \pm 0.01$ & $(4.6)$ & $0.27 \pm 0.02$ & $(2.4)$ & $0.35 \pm 0.03$ & (1.9) & $0.33 \pm 0.04$ & (4.0) \\
\hline $\mathrm{B} 2, \beta=0.4$ & $500.00 \pm 0.15$ & $(4.1)$ & $0.24 \pm 0.01$ & $(6.3)$ & $0.23 \pm 0.02$ & (7.6) & $0.04 \pm 0.02$ & $(1.2)$ & $0.75 \pm 0.01$ & (1.2) \\
\hline $\mathrm{B} 2, \beta=0.6$ & $500.00 \pm 0.14$ & $(3.6)$ & $0.26 \pm 0.01$ & $(5.5)$ & $0.24 \pm 0.02$ & $(6.4)$ & $0.07 \pm 0.02$ & $(1.1)$ & $0.65 \pm 0.02$ & (1.1) \\
\hline $\mathrm{B} 2, \beta=0.8$ & $500.00 \pm 0.16$ & $(4.7)$ & $0.22 \pm 0.01$ & $(5.6)$ & $0.24 \pm 0.03$ & (7.6) & $0.03 \pm 0.02$ & $(0.9)$ & $0.72 \pm 0.01$ & $(0.8)$ \\
\hline $\mathrm{B} 2, \beta=1.0$ & $500.00 \pm 0.14$ & $(3.9)$ & $0.10 \pm 0.01$ & $(2.5)$ & $0.23 \pm 0.02$ & $(7.1)$ & $0.01 \pm 0.02$ & $(0.6)$ & $0.86 \pm 0.01$ & $(0.6)$ \\
\hline $\mathrm{B} 1, \beta=0.01$ & $3.05 \pm 0.12$ & $(0.4)$ & $0.38 \pm 0.02$ & $(4.9)$ & $0.71 \pm 0.11$ & $(0.7)$ & $0.96 \pm 0.05$ & $(0.5)$ & $0.02 \pm 0.02$ & $(0.5)$ \\
\hline $\mathrm{B} 1, \beta=0.2$ & $24.09 \pm 0.08$ & $(1.2)$ & $0.28 \pm 0.02$ & (3.7) & $0.25 \pm 0.01$ & (1.4) & $0.46 \pm 0.03$ & (1.6) & $0.20 \pm 0.04$ & (3.7) \\
\hline $\mathrm{B} 1, \beta=0.4$ & $500.00 \pm 0.15$ & $(4.0)$ & $0.16 \pm 0.01$ & $(4.4)$ & $0.25 \pm 0.03$ & $(7.5)$ & $0.03 \pm 0.02$ & $(1.1)$ & $0.77 \pm 0.02$ & (1.0) \\
\hline $\mathrm{B} 1, \beta=0.6$ & $500.15 \pm 0.17$ & $(5.5)$ & $0.34 \pm 0.01$ & $(8.0)$ & $0.21 \pm 0.02$ & $(9.1)$ & $0.01 \pm 0.01$ & (1.2) & $0.81 \pm 0.01$ & (1.3) \\
\hline $\mathrm{B} 1, \beta=0.8$ & $500.17 \pm 0.17$ & $(5.4)$ & $0.34 \pm 0.01$ & $(8.1)$ & $0.25 \pm 0.03$ & $(8.5)$ & $2.21 \pm 0.02$ & (1.1) & $0.76 \pm 0.02$ & (1.1) \\
\hline $\mathrm{B} 1, \beta=1.0$ & $500.00 \pm 0.14$ & $(4.0)$ & $0.13 \pm 0.01$ & $(3.1)$ & $0.23 \pm 0.02$ & $(7.0)$ & $0.01 \pm 0.02$ & $(0.6)$ & $0.88 \pm 0.01$ & $(0.6)$ \\
\hline $\mathrm{A} 2, \beta=0.01$ & $2.93 \pm 0.24$ & $(1.7)$ & $0.34 \pm 0.02$ & $(6.4)$ & $0.83 \pm 0.23$ & $(2.2)$ & $0.99 \pm 0.01$ & (1.3) & $0.01 \pm 0.01$ & (1.3) \\
\hline $\mathrm{A} 2, \beta=0.2$ & $8.75 \pm 0.13$ & $(2.5)$ & $0.23 \pm 0.01$ & $(2.6)$ & $0.29 \pm 0.02$ & $(1.6)$ & $0.56 \pm 0.02$ & $(0.6)$ & $0.16 \pm 0.02$ & (1.4) \\
\hline $\mathrm{A} 2, \beta=0.4$ & $500.00 \pm 0.16$ & $(4.1)$ & $0.33 \pm 0.01$ & $(6.1)$ & $0.25 \pm 0.03$ & $(6.5)$ & $0.12 \pm 0.02$ & $(1.0)$ & $0.56 \pm 0.02$ & $(0.8)$ \\
\hline $\mathrm{A} 2, \beta=0.6$ & $500.00 \pm 0.14$ & $(3.4)$ & $0.17 \pm 0.01$ & $(4.2)$ & $0.26 \pm 0.03$ & $(5.7)$ & $0.14 \pm 0.03$ & $(1.2)$ & $0.52 \pm 0.02$ & $(0.9)$ \\
\hline $\mathrm{A} 2, \beta=0.8$ & $500.00 \pm 0.14$ & $(3.5)$ & $0.23 \pm 0.01$ & $(4.6)$ & $0.26 \pm 0.03$ & $(6.1)$ & $0.10 \pm 0.02$ & $(1.0)$ & $0.60 \pm 0.02$ & $(0.9)$ \\
\hline $\mathrm{A} 2, \beta=1.0$ & $500.00 \pm 0.16$ & $(4.2)$ & $0.21 \pm 0.01$ & $(3.9)$ & $0.26 \pm 0.03$ & $(5.2)$ & $0.13 \pm 0.03$ & $(1.3)$ & $0.57 \pm 0.02$ & (1.4) \\
\hline
\end{tabular}
fit. 

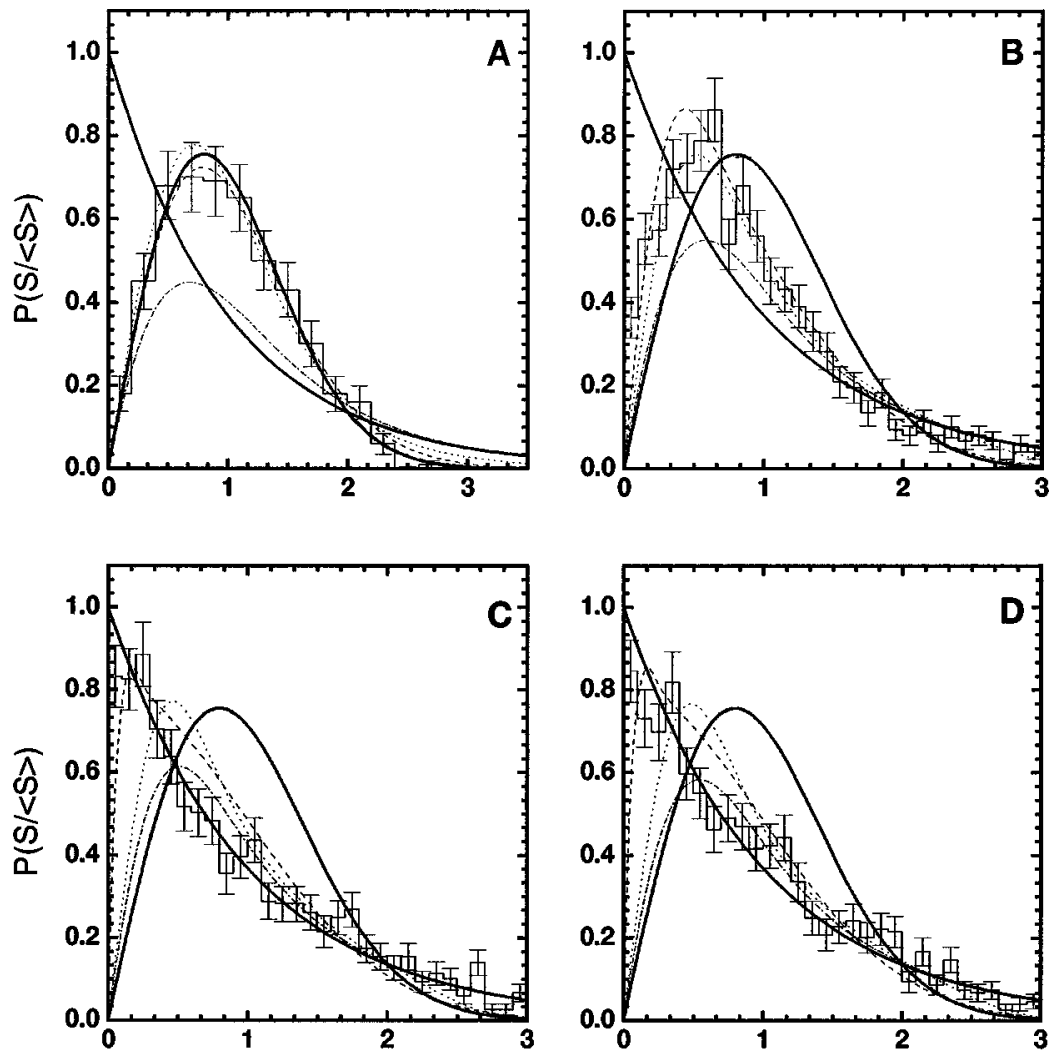

FIG. 1. (a)-(f) present the histograms of the NND's for the $B_{2}$ symmetry class and $\beta=0.01$, $0.20,0.40,0.60,0.80$, and 1.00 . The curves are the best fits from the DGOE (dashed), Caurier, Grammaticos, and Ramani (dot-dashed), and Lenz and Haake (dotted) models. Full curves show Poisson and GOE limiting cases.
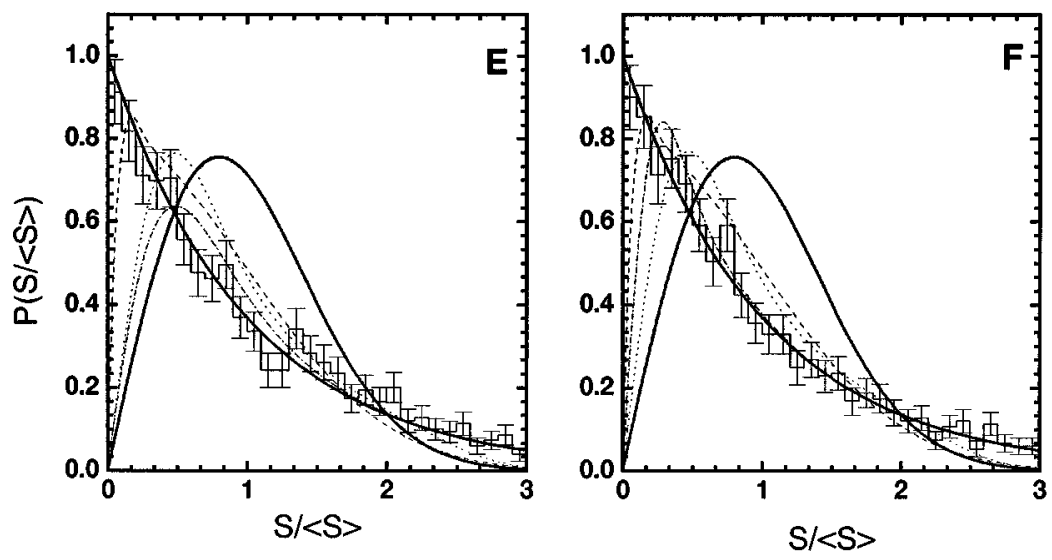

This result was used for the convergence analysis of the eigenvalues obtained numerically.

\section{ANALYSIS AND RESULTS}

The Hamiltonian was rotated by $\pi / 4$ in order to minimize the classically forbidden region of phase space and improve the convergence of the eigenvalues [16]. The symmetry classes of the system were also conveniently separated in order to simplify the identification of the level repulsion phenomena.

In this way, the eigenstates of the isotropic quartic oscillator can be classified according to the symmetry classes of the $C_{4 v}$ symmetry group. The irreducible representations of the group split into four one-dimensional representations and one two-dimensional representation (doubly degenerated). In this work we restricted ourselves to the four one-dimensional representations, usually labeled as $A_{1}$ (symmetric under reflections on the $x$ and $y$ axes, symmetric under reflections on the lines defined by $x+y=0$ and $x-y=0), B_{2}$ (antisymmetric, symmetric), $B_{1}$ (symmetric, antisymmetric), and $A_{2}$ (antisymmetric, antisymmetric).

The Hamiltonian was expanded in a convenient basis for each symmetry class, and the eigenvalues were numerically determined for different $\beta$ values. The frequency of the oscillator basis was chosen such as to minimize the trace of the Hamiltonian matrix from Eq. (23) and improve the convergence. In order to obtain a number of eigenvalues meaningful for the statistical analysis, matrices of dimension 3240 for the $A_{1}$ and $B_{2}$ symmetry classes and of dimension 3160 for $B_{1}$ and $A_{2}$ were constructed. The convergence of the eigenvalues was tested using relation (25) and requiring that the difference $N(E)-\overline{N(E)}$ must be very small.

Since Eq. (25) is only an approximation to $\overline{N(E)}$, the convergence test was done in a recursive way (this procedure is based on the arguments presented on Ref. [19]). In a first step, the eigenvalues were rescaled as 

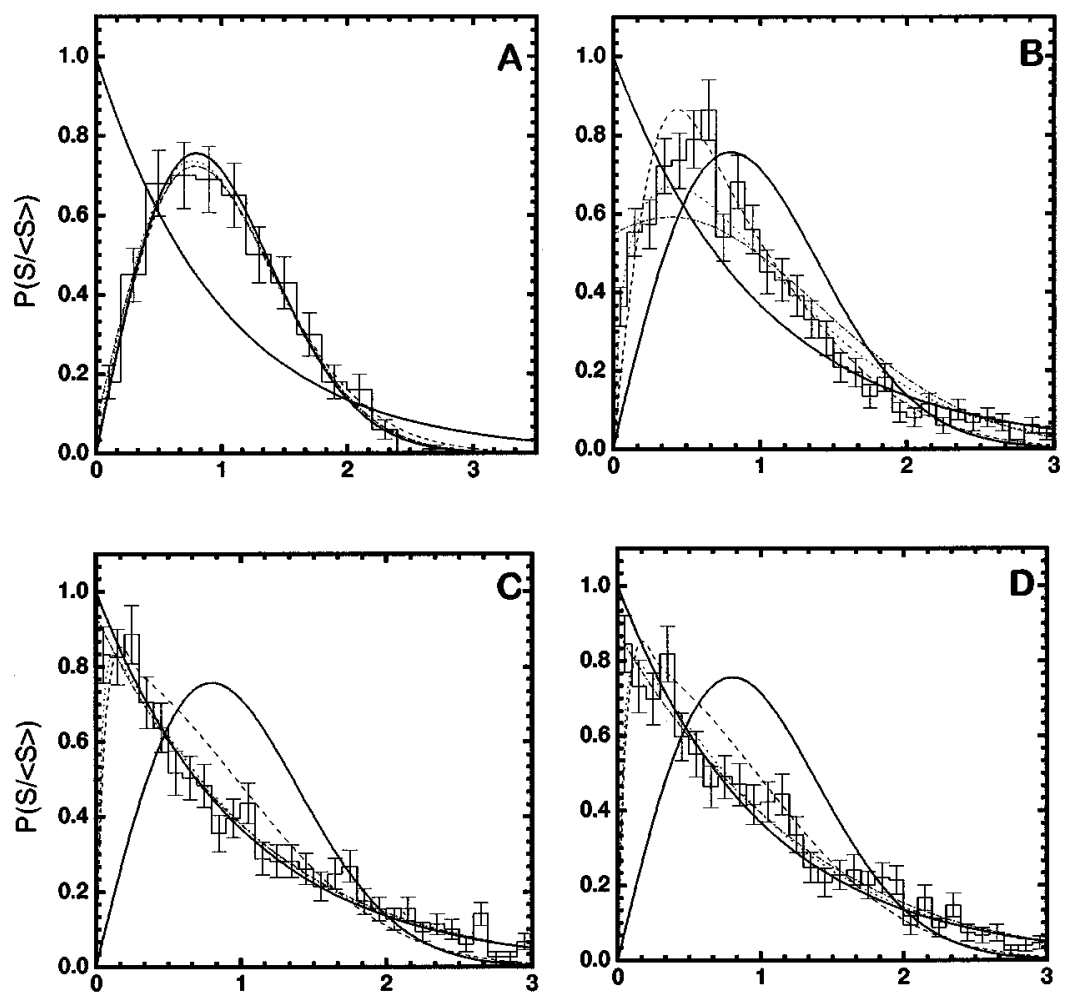

FIG. 2. The same as in Fig. 1, but for the DGOE (dashed), Brody (dotted), and Berry and Robnik (dotted-dashed) descriptions.
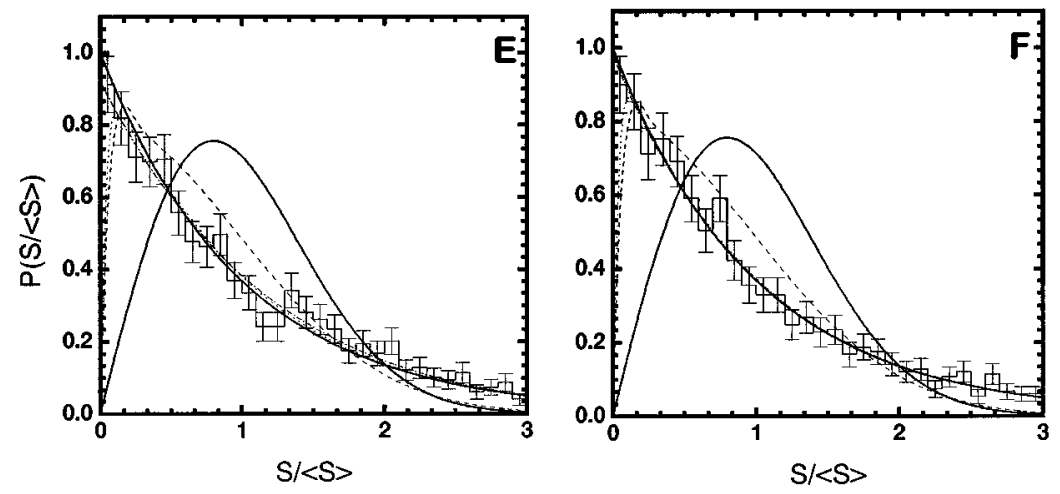

$$
E^{3 / 2} \rightarrow \varepsilon^{2}
$$

and $\overline{N(E)}$ was written as a function of $\varepsilon^{2}$. The mean staircase function was then supposed to be described by the exact version of Eq. (25). The differences between $N(E)$ and $\overline{N(E)}$ would then be caused by those eigenvalues with bad convergence. At the end of this first step there were 2100 converged eigenvalues for $\beta=0.01$, and 2300 for $\beta=0.20$, $0.40,0.60,0.80$, and 1.00 for the $A_{1}$ and $B_{2}$ symmetry classes; and 1800 for $\beta=0.01$, and 2100 for $\beta=0.20,0.40$, $0.60,0.80$, and 1.00 for $B_{1}$ and $A_{2}$.

Using just these "converged" eigenvalues, the calculations were repeated looking for the best fitted polynomial for $\overline{N\left(\varepsilon^{2}\right)}$. Since the mean staircase function is written in terms of $\varepsilon^{2}$, the degree of this polynomial could not be much larger than unity if the higher order terms in Eq. (25) were of lower relevance. In this case, higher degrees for this polynomial must be related to eigenvalues with bad convergence, which must be eliminated from the spectra for the analysis. All these steps were considered just in order to provide a more careful analysis of the convergence of the numerically obtained eigenvalues and to increase our confidence in the results.

In this way, 500 converged eigenvalues were obtained for $\beta=0.01$, and 1500 for $\beta=0.20,0.40,0.60,0.80$, and 1.00 for the $A_{1}$ and $B_{2}$ symmetry classes; and 450 for $\beta=0.01$, and 1400 for $\beta=0.20,0.40,0.60,0.80$, and 1.00 for $B_{1}$ and $A_{2}$. The degree of the polynomials considered for the unfolding were 1,2 , or 3 , according to the symmetry class and $\beta$ value.

The NND's obtained from the unfolded converged eigenvalues were then analyzed using the five formulas described above and normalized $\chi^{2}$ values were calculated for the fittings in the usual way. Possible effects on these $\overline{\chi^{2}}$ values due to a smaller number of eigenvalues were also investigated. In this case, cutting some hundreds of the higher or lower eigenvalues of the spectra showed no other effects than the statistical fluctuations related to the different amount of data.

The limiting parameter values used in the numerical calculations-those for which the five distributions reproduce the Wigner and Poisson cases-are displayed in Table 

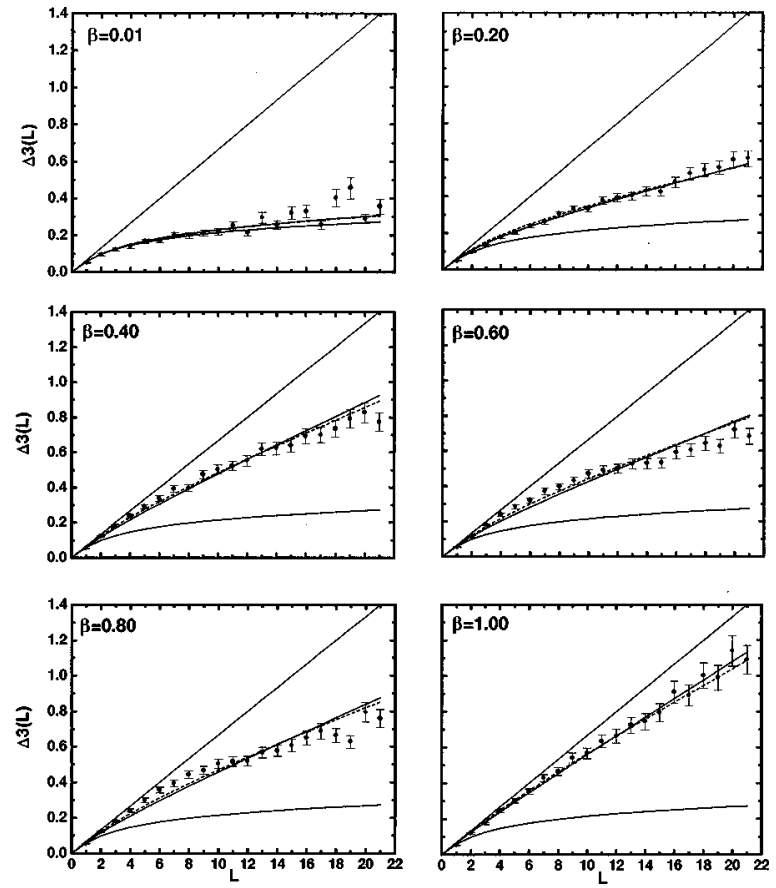

FIG. 3. Dyson-Mehta statistic for the $B_{2}$ symmetry class and $\beta=0.01,0.20,0.40,0.60,0.80$, and 1.00. The best fits from the Berry-Robnik expression (10) (dashed) and the ansatz (21) (varying full curve) are given. The uppermost and the lowermost full curves correspond to the limiting cases of Poisson and GOE statistics, respectively.

I. The five numerically adjusted parameters for each $\beta$ value for each symmetry class are presented in Table II. The curves and histograms for the NND's for all analyzed $\beta$ values for the $B_{2}$ symmetry class are displayed in Figs.
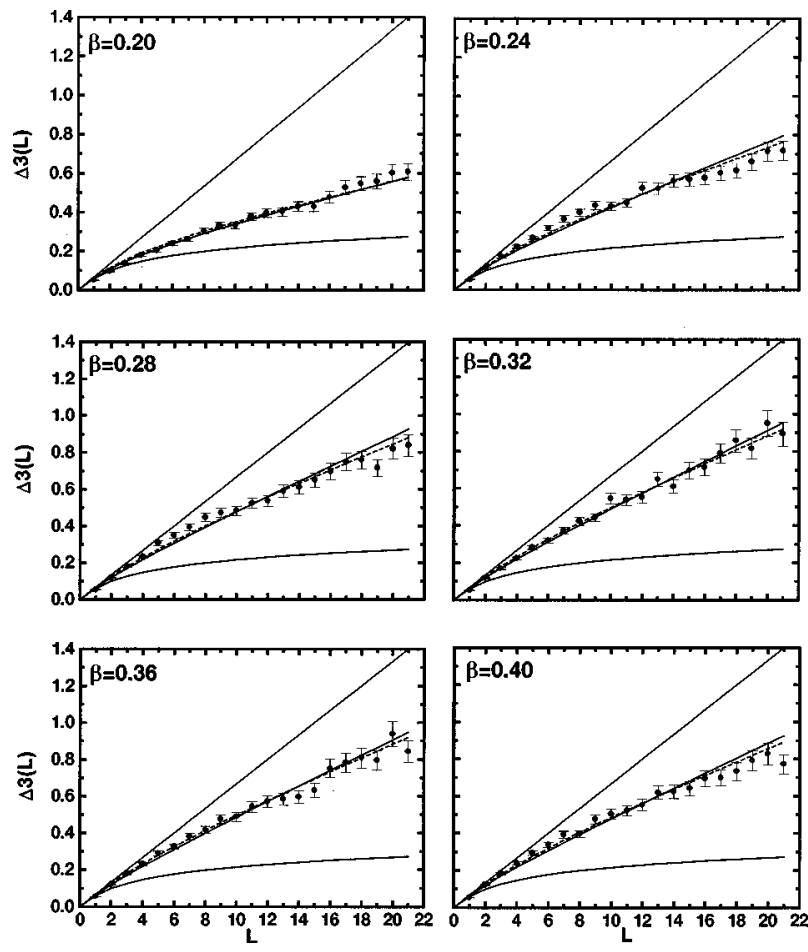

FIG. 4. The same as in Fig. 3, but for $\beta=0.20,0.24,0.28,0.32$, 0.36 , and 0.40 .
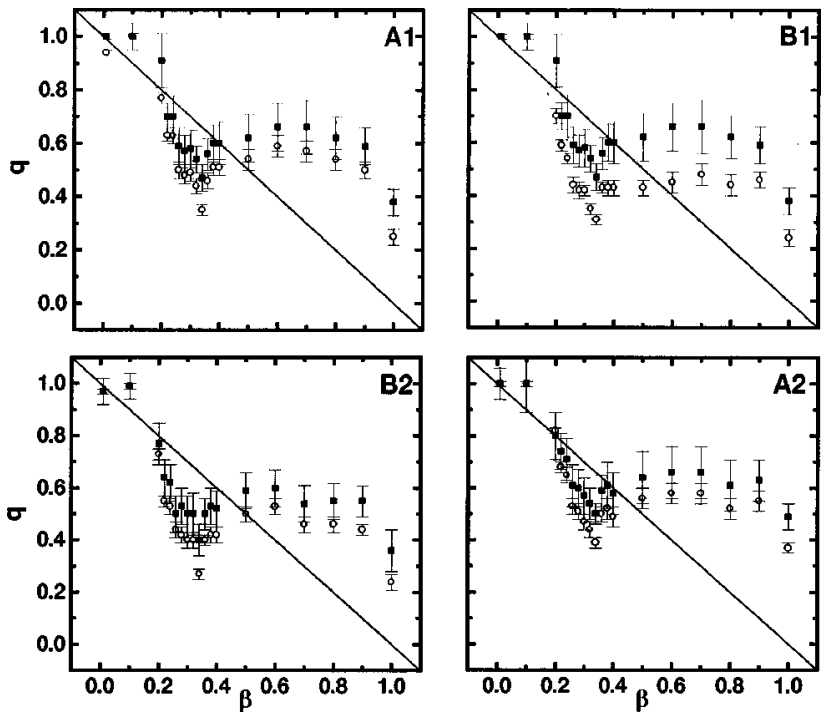

FIG. 5. Adjusted $q$ parameters for ansatz (21) (open circle) and the Berry-Robnik expression (10) (full square) for the $A_{1}, B_{1}, B_{2}$, and $A_{2}$ symmetry classes.

$1(\mathrm{~A})-1(\mathrm{~F})$ and $2(\mathrm{~A})-2(\mathrm{~F})$ (the results for the other three symmetry classes are similar).

The rich structure seen in the phase space analysis of the transition between chaos and order of the quartic oscillator is not observed here [20]. A possible explanation may be that the quantum calculation hides, due to the linear nature of the Schrödinger equation, the classical phase space structure. Additionally, we would like to point out that statistical data based analyses are not in a position to show all the details present in the transition between order and chaos. Rather, after an appropriate rescaling of variables, the random matrix theory would be able to describe some universal statistical properties shared by a large class of Hamiltonians [21].

The results obtained with expression (10) and ansatz (21) for $\Delta_{3}(L)$ are shown in Figs. 3 and 4. The parameters of these two distributions were both called $q$ in order to simplify the notation: in both cases, the Poissonian limit corresponds to $q=0$ and 1 is the Wigner distribution. The BerryRobnik parameter is once more a measure of the chaotic fraction of phase space. Figure 5 displays the adjusted $q$ parameters for Eqs. (10) and (21) as a function of the $\beta$ value of the oscillator [Eq. (23)] for the four analyzed symmetry classes.

The first point worth mentioning is the apparent absence of level repulsion for intermediate $\beta$ values in the histograms of Figs. 1 and 2. This is a consequence of the relatively small number of eigenvalues considered due to computational constraints.

In Figs. 3 and $4, \Delta_{3}(L)$ shows that short and long range correlations undergo a transition between chaos and order for increasing $\beta$ values with different speeds: the most regular situation is achieved much faster by short range correlations than by long range correlations. This result is an interesting aspect of the transition between chaos and order of the quartic oscillator that we are not able to explain.

According to the NND's, we note the good agreement between the results of all five descriptions concerning the chaoticity of the system: with increasing $\beta$ the quantized quartic oscillator becomes more and more regular, as ex- 
pected. Nevertheless, it is interesting to see that the most regular value of the CGR, $\mathrm{LH}$, and BR parameters are never achieved even if the system is completely regular $(\beta$ $=1.0$ ). In the DGOE case, the Poissonian curve is obtained when $\alpha \rightarrow \infty$. Here, the maximum parameter value obtained is already a good result, as shown by the $\overline{\chi^{2}}$ values displayed in Table II.

The most Poissonian case of the DGOE model displays a kind of "shoulder" for small $s /\langle s\rangle$ values. This can be explained by a simple physical argument: as soon as the level repulsion is introduced in the system, degeneracies are lifted, causing a small increase in the probability for very small spacings between levels. This fact together with the normalization constraint of the distribution causes the shoulder in the DGOE curve for nearly Poissonian parameter values [22]. Unfortunately, this and other aspects of the NND's for small spacings can only be observed in histograms obtained from a higher amount of data, not available here.

Note the saturation of the adjusted DGOE parameter: already for $\beta=0.4$, the maximum parameter value is obtained. This is probably related to the absence of level repulsion in the intermediate situation between maximum chaos and maximum regularity - seen in the histograms of NND—due to the relative small number of eigenvalues considered, as already pointed out. It is also possible to see a saturation for the LH parameter for $\beta \geqslant 0.4$ and this behavior shows up in all other three symmetry classes.

By inspection of Fig. 1(A), it is possible to see problems in the CGR description of data: the adjusted curve lies far below the histogram, what is also represented in Table II by the $\overline{\chi^{2}}$ values for $\beta=0.01$. In fact, it hardly provides a good description in all cases except that of $\beta=1.0$ (strongly Poissonian situation). This is probably due to the strong approximations introduced by the authors of Ref. [5] in the derivation of Eq. (6).

According to the $\overline{\chi^{2}}$ values, the LH distribution has problems describing the data with increasing regularity of the system. The difficulties with the numerical fitting increase as $\lambda \rightarrow 0$, i.e., the Poissonian limit [see Eq. (8)], and were already noted by another group [23]. Here we tested different numerical methods to calculate the special functions and related integrals in Eq. (8) and used those which gave the most stable results (subroutines GAULEG and TRAPZD from Ref. [24]).

The $\overline{\chi^{2}}$ results displayed in Table II show smaller values for the Brody distribution, the BR case, and the DGOE.

Concerning the Dyson-Mehta statistic displayed in Figs. 3 and 4 , it is interesting to note the good agreement between the two descriptions tested: in almost all cases it is even difficult to distinguish between the two curves. This is prob- ably due to the $\Delta_{3}^{\mathrm{Poi}}(L)$ term [Eq. (11)], which is the same for Eqs. (10) and (21) and seems to dominate the behavior of both curves. Also, the results from Fig. 5 show a peculiar relation between the adjusted $\Delta_{3}(L)$ parameters and the $\beta$ values [Eq. (23)]. Such behavior is related to the different chaos-order transition speeds for the short and long range correlations between eigenvalues, as already mentioned above.

\section{FINAL REMARKS}

In this work, five formulas proposed to describe systems in the transition region between chaos and order were compared, namely, the Brody ansatz, the Berry-Robnik model, the Caurier-Grammaticos-Ramani model, the Lenz-Haake model, and the DGOE model. In order to do this, the five proposed NND's were applied to the spectra of the isotropic quartic oscillator.

The long range counterpart of the level correlations were analyzed in terms of the Dyson-Mehta statistic $\Delta_{3}$. Here an expression usually associated with the Berry-Robnik formalism was applied. A simple expression for the $\Delta_{3}$ statistic which interpolates between the Poissonian and GOE limits was also considered. The results show that the short and long range correlations undergo a transition between chaos and order for increasing $\beta$ value of the quartic oscillator [Eq. (23)] with different speeds.

In all cases, the NND of the DGOE provided a good description for the level fluctuations of the spectra. The comparison of the models teaches us that the DGOE model has advantages over the others: (i) it provides as good a description for the NND as any one of the other four formulas; and (ii) it provides an analytical expression for the probability distribution of the $N \times N$ matrix ensemble, which allows the study of the statistical fluctuation properties of the elements of the eigenvectors (as already done in Ref. [15]), and also opens a new possibility of investigation of the long range correlations among levels.

\section{ACKNOWLEDGMENTS}

The authors would like to thank the LCCA/CCE-USP (São Paulo, SP, Brazil), for the use of its facilities. One of us (C. I. B.) wishes to thank H. L. Harney for helpful criticisms concerning this manuscript. The research was supported in part by CNPq (Conselho Nacional de Desenvolvimento Científico e Tecnológico, Brazil), and FAPESP (Fundação de Amparo à Pesquisa do Estado de São Paulo, Brazil). The work of C. I. Barbosa was supported in part by CAPES (Coordenação de Aperfeiçoamento do Pessoal de Ensino Superior, Brazil), FAPESP, and Fritz ThyssenStiftung (Germay).
[1] M. V. Berry and M. Tabor, Proc. R. Soc. London, Ser. A 356, 375 (1977).

[2] O. Bohigas, M. -J. Giannoni, and C. Schmit, Phys. Rev. Lett. 52, 1 (1984).
[3] O. Bohigas, in Proceedings of the Les Houches Summer School on Chaos and Quantum Physics, Les Houches, 1989, edited by M.-J. Giannoni, A. Voros, and J. Zinn-Justin (NorthHolland, Amsterdam, 1991), p. 89. 
[4] M. V. Berry and M. Robnik, J. Phys. A 17, 2413 (1984).

[5] E. Caurier, B. Grammaticos, and A. Ramani, J. Phys. A 23, 4903 (1990).

[6] G. Lenz and F. Haake, Phys. Rev. Lett. 67, 1 (1991).

[7] F. J. Dyson, J. Math. Phys. 3, 140 (1962).

[8] M. S. Hussein and M. P. Pato, Phys. Rev. Lett. 70, 1089 (1993).

[9] T. A. Brody, Lett. Nuovo Cimento 7, 482 (1973).

[10] T. A. Brody, J. Flores, J. B. French, P. A. Mello, A. Pandey, S. S. M. Wong, Rev. Mod. Phys. 53, 385 (1981).

[11] I. S. Gradshteyn and I. M. Ryzhik, Table of Integrals, Series and Products (Academic, New York, 1965).

[12] H. Alt, H.-D. Gräf, H. L. Harney, R. Hofferbert, H. Lengeler, C. Rangacharyulu, A. Richter, and P. Schardt, Phys. Rev. E 50, R1 (1994).

[13] R. Balian, Nuovo Cimento B 57, 183 (1968).

[14] M. P. Pato, C. A. Nunes, C. L. Lima, M. S. Hussein, and Y. Alhassid, Phys. Rev. C 49, 2919 (1994).
[15] M. S. Hussein and M. P. Pato, Phys. Rev. C 47, 2401 (1993).

[16] B. Eckhardt, G. Hose, and E. Pollak, Phys. Rev. A 39, 3776 (1989).

[17] S. G. Mantinyan, G. K. Savvidy, and N. G. TerArutyunyuanSavvidy, Zh. Éksp. Teor. Fiz. 80, 831 (1981) [Sov. Phys. JETP 53, 421 (1981)].

[18] G. K. Savvidy, Nucl. Phys. B 246, 302 (1984).

[19] O. Bohigas, S. Tomsovic, and D. Ullmo, Phys. Rep. 223, 43 (1993).

[20] T. H. Seligman, J. J. M. Verbaarschot, and M. R. Zirnbauer, J. Phys. A 18, 2751 (1985).

[21] M. S. Hussein and M. P. Pato, Phys. Rev. Lett. 80, 1003 (1998).

[22] T. Guhr, Ann. Phys. (N.Y.) 250, 145 (1996).

[23] H. Alt and R. Hofferbert (private communication).

[24] W. H. Press, S. A. Teukolsky, W. T. Vetterling, and B. P. Flannery, Numerical Recipes in Fortran-the Art of Scientific Computing (Cambridge University Press, New York, 1992). 